\title{
Exact Solutions for Unsteady Free Convection Flow of Casson Fluid over an Oscillating Vertical Plate with Constant Wall Temperature
}

\author{
Asma Khalid, ${ }^{1,2}$ Ilyas Khan, ${ }^{3}$ and Sharidan Shafie ${ }^{1}$ \\ ${ }^{1}$ Department of Mathematical Sciences, Faculty of Science, Universiti Teknologi Malaysia (UTM), 81310 Skudai, Malaysia \\ ${ }^{2}$ Department of Mathematics, SBK Women's University, Quetta 87300, Pakistan \\ ${ }^{3}$ College of Engineering, Majmaah University, Majmaah 11952, Saudi Arabia \\ Correspondence should be addressed to Sharidan Shafie; sharidan@utm.my
}

Received 17 July 2014; Accepted 23 October 2014

Academic Editor: Yasir Khan

Copyright (C) 2015 Asma Khalid et al. This is an open access article distributed under the Creative Commons Attribution License, which permits unrestricted use, distribution, and reproduction in any medium, provided the original work is properly cited.

\begin{abstract}
The unsteady free flow of a Casson fluid past an oscillating vertical plate with constant wall temperature has been studied. The Casson fluid model is used to distinguish the non-Newtonian fluid behaviour. The governing partial differential equations corresponding to the momentum and energy equations are transformed into linear ordinary differential equations by using nondimensional variables. Laplace transform method is used to find the exact solutions of these equations. Expressions for shear stress in terms of skin friction and the rate of heat transfer in terms of Nusselt number are also obtained. Numerical results of velocity and temperature profiles with various values of embedded flow parameters are shown graphically and their effects are discussed in detail.
\end{abstract}

\section{Introduction}

Newtonian fluids described by Navier-Stokes equations are extensively studied in the literature for the past few decades. Largely, this is due to the fact that they are relatively simple and their solutions are convenient [1-10]. However, Newtonian fluids which have a linear relationship between the stress and the rate of strain are limited in view of their applications. They do not explain several phenomena observed for the fluids in industry and other technological applications. For example, many complex fluids such as blood, soap, clay coating, certain oils and greases, elastomers, suspensions, and many emulsions are noteworthy due to their various applications in industry. Unfortunately, Navier-Stokes equations are no more convincing to describe such fluids. In the literature, they are known as non-Newtonian fluids. These fluids are described by a nonlinear relationship between the stress and the rate of strain. The understanding of flow characteristics of non-Newtonian fluids is very important because they play a significant role in industry and engineering. Such interest is motivated because of extensive applications in diverse areas of biorheology, geophysics, and chemical and petroleum industries [11, 12]. In view of these particular applications, the study and understanding of non-Newtonian fluids have now become an increasingly appealing topic of current research in this field.

Rheological properties of non-Newtonian fluids are described by their so-called constitutive equations. Due to complexity of fluids, several non-Newtonian fluid models/constitutive equations based on their empirical observations have been proposed. Amongst the different nonNewtonian fluids there is one known as Casson fluid which was originally introduced by Casson for the prediction of the flow behavior of pigment-oil suspensions [13]. The Casson model is based on a structure model of the interactive behavior of solid and liquid phases of a two-phase suspension. Casson fluid exhibits yield stress. It is a shear thinning liquid which has an infinite viscosity at zero rates of shear, a yield stress below which no flow occurs, and a zero viscosity at an infinite rate of shear [14]. More exactly, if a shear stress less than the yield stress is applied to the fluid, it behaves like a solid, whereas if a shear stress greater than yield 
stress is applied, it starts to move. Some famous examples of Casson fluid include jelly, tomato sauce, honey, soup, and concentrated fruit juices. Human blood can also be treated as Casson fluid due to the presence of several substances such as protein, fibrinogen, and globulin in aqueous base plasma and human red blood cells $[15,16]$.

In the earlier studies on Casson fluid, Fredrickson [17] investigated its steady flow in a tube whereas Boyd et al. [18] described the steady and oscillatory flow of blood flow by taking into account Casson fluid. Mernone et al. [19] discussed the peristaltic flow of a Casson fluid in a twodimensional channel. Mustafa et al. [20] studied the unsteady boundary layer flow and heat transfer of a Casson fluid over a moving flat plate with a parallel free stream using homotopy analysis method (HAM). Mixed convection stagnation-point flow of Casson fluid with convective boundary conditions is examined by Hayat et al. [21]. Mukhopadhyay [22] described the effects of thermal radiation on Casson fluid flow and heat transfer over an unsteady stretching surface subjected to suction/blowing. Mukhopadhyay et al. [23] also analyzed the Casson fluid flow over an unsteady stretching surface. Bhattacharyya [24] investigated the boundary layer stagnationpoint flow of Casson fluid and heat transfer towards a shrinking/stretching sheet and Pramanik [25] studied the Casson fluid flow and heat transfer past an exponentially porous stretching surface in presence of thermal radiation.

In all of the above studies the solutions of Casson fluid are obtained by using either approximate method or any numerical scheme. There are very few cases in which the exact analytical solutions of Casson fluid are obtained. These solutions are even rare when Casson fluid in free convection flow with constant wall temperature is considered. On the other hand, the flow of Casson fluids (such as drilling muds, clay coatings and other suspensions, certain oils and greases, polymer melts, blood, and many emulsions), in the presence of heat transfer, is an important research area due to its relevance to the optimized processing of chocolate, toffee, and other foodstuffs [26].

Motivated by the above investigations, the present analysis is focused on the study of unsteady boundary layer flow of a Casson fluid past an oscillating vertical plate with constant wall temperature. Exact solutions are obtained by using the Laplace transform technique. Analytical as well as numerical results for skin friction and Nusselt number are provided. Graphical results are presented and discussed for various physical parameters. Exact solutions obtained in this paper are important; not only do they correspond to some fundamental flow situations, but also they are useful for explaining the flow physics in detail as well as for being used as a benchmark for validation of other solutions obtained via approximate or numerical schemes.

\section{Formulation of the Problem}

Let us consider the effect of heat transfer on unsteady boundary layer flow of an incompressible Casson fluid past an infinite vertical flat plate situated at the flow being confined to $y>0$, where $y$ is the coordinate measured in the normal direction to the surface. It is assumed that, at the initial

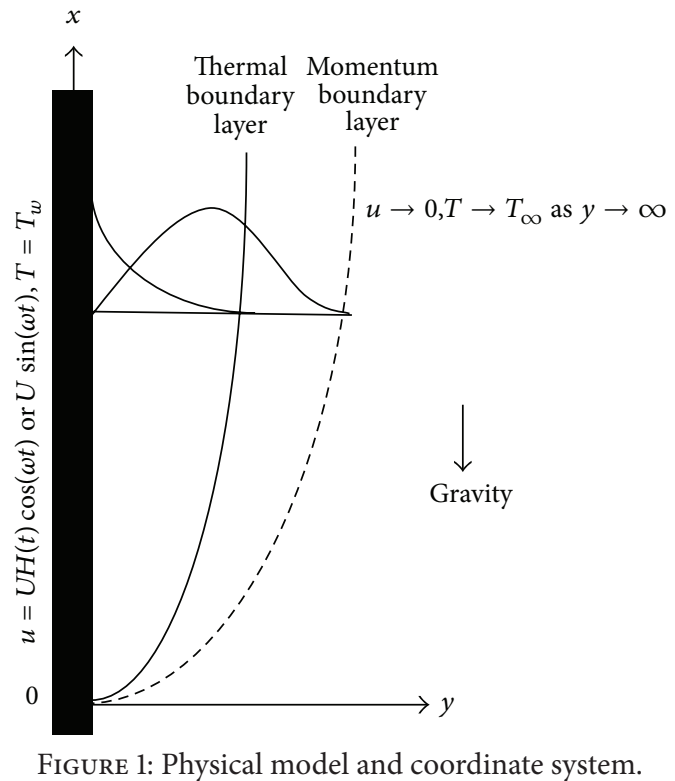

moment $t=0$, both the plate and fluid are at rest with constant temperature $T_{\infty}$. At time $t=0^{+}$the plate begins to oscillate in its plane $(y=0)$ according to

$$
\mathbf{V}=U H(t) \cos (\omega t) \mathbf{i} ; \quad \text { or } \quad \mathbf{V}=U \sin (\omega t) \mathbf{i} ; \quad t>0,
$$

where the constant $U$ is the amplitude of the plate oscillations, $H(t)$ is the unit step function, $\mathbf{i}$ is the unit vector in the vertical flow direction, and $\omega$ is the frequency of oscillation of the plate. At the same time, the plate temperature is raised to $T_{w}$ which is thereafter maintained constant (Figure 1).

We assume that the rheological equation of state for an isotropic and incompressible flow of a Casson fluid can be written as (see the study by Mukhopadhyay [22])

$$
\tau=\tau_{0}+\mu \gamma^{\bullet}
$$

or

$$
\tau_{i j}= \begin{cases}2\left(\mu_{B}+\frac{p_{y}}{\sqrt{2 \pi}}\right) e_{i j}, & \pi>\pi_{c} \\ 2\left(\mu_{B}+\frac{p_{y}}{\sqrt{2 \pi_{c}}}\right) e_{i j}, & \pi<\pi_{c},\end{cases}
$$

where $\pi=e_{i j} e_{i j}$ and $e_{i j}$ is the $(i, j)^{i h}$ component of the deformation rate, $\pi$ is the product of the component of deformation rate with itself, $\pi_{c}$ is a critical value of this product based on the non-Newtonian model, $\mu_{B}$ is plastic dynamic viscosity of the non-Newtonian fluid, and $p_{y}$ is yield stress of fluid. Under these conditions along with the assumption that the viscous dissipation term in the energy equation is neglected, we get the following set of partial differential equations:

$$
\begin{gathered}
\rho \frac{\partial u}{\partial t}=\mu_{B}\left(1+\frac{1}{\gamma}\right) \frac{\partial^{2} u}{\partial y^{2}}+\rho g \beta\left(T-T_{\infty}\right), \\
\rho c_{p} \frac{\partial T}{\partial t}=k \frac{\partial^{2} T}{\partial y^{2}}
\end{gathered}
$$


together with initial and boundary conditions

$$
\begin{gathered}
t<0: u=0, \quad T=T_{\infty} \quad \forall y>0, \\
t \geq 0: u=U H(t) \cos (\omega t) \quad \text { or } \quad u=U \sin (\omega t), \\
T=T_{w} \quad \text { at } y=0, \\
u \longrightarrow 0, \quad T \longrightarrow T_{\infty} \quad \text { as } y \longrightarrow \infty .
\end{gathered}
$$

We introduce the dimensionless variables

$$
\begin{gathered}
u^{*}=\frac{u}{U}, \quad y^{*}=\frac{U}{v} y, \quad t^{*}=\frac{U^{2}}{v} t \\
\theta=\frac{T-T_{\infty}}{T_{w}-T_{\infty}}, \quad \omega^{*}=\frac{\omega \nu}{U^{2}}, \quad \tau^{*}=\frac{\tau}{\rho u^{2}}
\end{gathered}
$$

into (4)-(5), and we get (* symbols are dropped for simplicity)

$$
\begin{gathered}
\frac{\partial u}{\partial t}=\left(1+\frac{1}{\gamma}\right) \frac{\partial^{2} u}{\partial y^{2}}+\mathrm{Gr} \theta \\
\operatorname{Pr} \frac{\partial \theta}{\partial t}=\frac{\partial^{2} \theta}{\partial y^{2}}
\end{gathered}
$$

with associated initial and boundary conditions

$$
\begin{gathered}
t<0: u=0, \quad \theta=0 \quad \forall y>0, \\
t \geq 0: u=H(t) \cos (\omega t) \quad \text { or } \quad u=\sin (\omega t), \\
\theta=1 \quad \text { at } y=0, \\
u \longrightarrow 0, \quad \theta \longrightarrow 0 \quad \text { as } y \longrightarrow \infty,
\end{gathered}
$$

where

$$
\operatorname{Pr}=\frac{\mu c_{p}}{k}, \quad \mathrm{Gr}=\frac{\nu g \beta\left(T_{w}-T_{\infty}\right)}{U^{3}}, \quad \gamma=\frac{\mu_{B} \sqrt{2 \pi_{c}}}{p_{y}}
$$

are the Prandtl, Grashof numbers, and the Casson parameter.

\section{Solution of the Problem}

Applying Laplace transforms to (7) and (8), using initial and boundary conditions (9), we get the following solutions in the transformed $(y, q)$ plane:

$$
\begin{gathered}
\bar{\theta}(y, q)=\frac{1}{q} e^{-y \sqrt{\operatorname{Pr} q}}, \\
\bar{u}(y, q)=\frac{q}{q^{2}+\omega^{2}} e^{-y \sqrt{a q}}+\frac{b}{q^{2}} e^{-y \sqrt{a q}}+\frac{b}{q^{2}} e^{-y \sqrt{\operatorname{Pr} q}} .
\end{gathered}
$$

The inverse Laplace transforms of (11) and (12) are obtained as follows:

$$
\theta(y, t)=\operatorname{erf} c\left(\frac{y}{2} \sqrt{\frac{\operatorname{Pr}}{t}}\right)
$$

$u_{c}(y, t)$

$$
=\frac{H(t)}{4} e^{-i \omega t}\left[e^{-y \sqrt{-i \omega a}} \operatorname{erf} c\left(\frac{y}{2} \sqrt{\frac{a}{t}}-\sqrt{-i \omega t}\right)\right.
$$

$$
\begin{gathered}
\left.+e^{y \sqrt{-i \omega a}} \operatorname{erf} c\left(\frac{y}{2} \sqrt{\frac{a}{t}}+\sqrt{-i \omega t}\right)\right] \\
+\frac{H(t)}{4} e^{i \omega t}\left[e^{-y \sqrt{i \omega a}} \operatorname{erf}\left(\frac{y}{2} \sqrt{\frac{a}{t}}-\sqrt{i \omega t}\right)\right. \\
\left.+e^{y \sqrt{i \omega a}} \operatorname{erf} c\left(\frac{y}{2} \sqrt{\frac{a}{t}}+\sqrt{+i \omega t}\right)\right] \\
+b\left[\left(t+\frac{a y^{2}}{2}\right) \operatorname{erf} c\left(\frac{y}{2} \sqrt{\frac{a}{t}}\right)-y \sqrt{a} \sqrt{\frac{t}{\pi}} e^{-a y^{2} / 4 t}\right] \\
-b\left[\left(t+\frac{\operatorname{Pr} y^{2}}{2}\right) \operatorname{erf} c\left(\frac{y}{2} \sqrt{\frac{\operatorname{Pr}}{t}}\right)\right. \\
\left.-y \sqrt{\operatorname{Pr}} \sqrt{\frac{t}{\pi}} e^{-\operatorname{Pr} y^{2} / 4 t}\right] .
\end{gathered}
$$

The subscript " $c$ " on the left side of (14) stands for the cosine oscillations of the plate. Similarly, the velocity corresponding to the sine oscillations of the plate is given by

$$
\begin{aligned}
& u_{s}(y, t) \\
& =-\frac{1}{4 i} e^{-i \omega t} \\
& \times\left[e^{-y \sqrt{-i \omega a}} \operatorname{erf} c\left(\frac{y}{2} \sqrt{\frac{a}{t}}-\sqrt{-i \omega t}\right)\right. \\
& \left.+e^{y \sqrt{-i \omega a}} \operatorname{erf} c\left(\frac{y}{2} \sqrt{\frac{a}{t}}+\sqrt{-i \omega t}\right)\right] \\
& +\frac{1}{4 i} e^{i \omega t}\left[e^{-y \sqrt{i \omega a}} \operatorname{erf} c\left(\frac{y}{2} \sqrt{\frac{a}{t}}-\sqrt{i \omega t}\right)\right. \\
& +b\left[\left(t+\frac{a y^{2}}{2}\right) \operatorname{erf} c\left(\frac{y}{2} \sqrt{\frac{a}{t}}\right)-y \sqrt{a} \sqrt{\frac{t}{\pi}} e^{-a y^{2} / 4 t}\right. \\
& -b\left[\left(t+\frac{\operatorname{Pr} y^{2}}{2}\right) \operatorname{erf} c\left(\frac{y}{2} \sqrt{\frac{a}{t}}+\sqrt{\frac{\operatorname{Pr}}{t}}\right)\right. \\
& \left.-y \sqrt{\operatorname{Pr}} \sqrt{\frac{t}{\pi}} e^{-\operatorname{Pr} y^{2} / 4 t}\right] \\
& +
\end{aligned}
$$


where

$$
a=\frac{\gamma}{1+\gamma}, \quad b=\frac{a \mathrm{Gr}}{\operatorname{Pr}-1} .
$$

Note that the above solutions for velocity are only valid for $\operatorname{Pr} \neq 1$. Moreover the solution for $\operatorname{Pr}=1$ can be easily obtained by putting $\operatorname{Pr}=1$ into (8) and can follow a similar procedure as discussed above. The obtained solutions for cosine and sine oscillations of the plate when $\operatorname{Pr}=1$ are

$$
\begin{aligned}
& u_{c}(y, t) \\
& =\frac{H(t)}{4} e^{-i \omega t} \\
& \times\left[e^{-y \sqrt{-i \omega a}} \operatorname{erf} c\left(\frac{y}{2} \sqrt{\frac{a}{t}}-\sqrt{-i \omega t}\right)\right. \\
& \left.+e^{y \sqrt{-i \omega a}} \operatorname{erf} c\left(\frac{y}{2} \sqrt{\frac{a}{t}}+\sqrt{-i \omega t}\right)\right] \\
& +\frac{H(t)}{4} e^{i \omega t}\left[e^{-y \sqrt{i \omega a}} \operatorname{erf} c\left(\frac{y}{2} \sqrt{\frac{a}{t}}-\sqrt{i \omega t}\right)\right. \\
& \left.+e^{y \sqrt{i \omega a}} \operatorname{erf} c\left(\frac{y}{2} \sqrt{\frac{a}{t}}+\sqrt{+i \omega t}\right)\right] \\
& +\frac{a \mathrm{Gr} y}{2}\left[2 \sqrt{\frac{t}{\pi}} e^{-y^{2} / 4 t}-y \operatorname{erf} c\left(\frac{y}{2 \sqrt{t}}\right)\right], \\
& u_{s}(y, t) \\
& =-\frac{1}{4 i} e^{-i \omega t} \\
& \times\left[e^{-y \sqrt{-i \omega a}} \operatorname{erf} c\left(\frac{y}{2} \sqrt{\frac{a}{t}}-\sqrt{-i \omega t}\right)\right. \\
& \left.+e^{y \sqrt{-i \omega a}} \operatorname{erf} c\left(\frac{y}{2} \sqrt{\frac{a}{t}}+\sqrt{-i \omega t}\right)\right] \\
& +\frac{1}{4 i} e^{i \omega t}\left[e^{-y \sqrt{i \omega a}} \operatorname{erf} c\left(\frac{y}{2} \sqrt{\frac{a}{t}}-\sqrt{i \omega t}\right)\right. \\
& \left.+e^{y \sqrt{i \omega a}} \operatorname{erf} c\left(\frac{y}{2} \sqrt{\frac{a}{t}}+\sqrt{+i \omega t}\right)\right] \\
& +\frac{a \mathrm{Gr} y}{2}\left[2 \sqrt{\frac{t}{\pi}} e^{-y^{2} / 4 t}-y \operatorname{erf} c\left(\frac{y}{2 \sqrt{t}}\right)\right] .
\end{aligned}
$$

Note that, in (14) and (15), the first two terms in each equation account for the contribution from mechanical parts while the last two terms show the thermal effects. On the other hand in (17) and (18), the last term in each equation shows the contribution from the thermal part.
The nondimensional skin friction is calculated from the velocity field (14), using the relation

$$
\begin{gathered}
\tau=-\left.\mu\left(1+\frac{1}{\gamma}\right) \frac{\partial u}{\partial y}\right|_{y=0}, \\
\tau=\frac{1}{2 a \sqrt{\pi}} \\
\times\left[e ^ { - i t \omega } \left[2 e ^ { i t \omega } \left\{H \sqrt{\frac{a}{t}}+\sqrt{a} b \sqrt{t}-a b \sqrt{\operatorname{Pr}} \sqrt{t}\right.\right.\right. \\
\left.+b \sqrt{\frac{a}{t} t+a b} \sqrt{\frac{\operatorname{Pr}}{t} t}\right\} \\
+H \sqrt{\pi}\{\sqrt{-i a \omega} \operatorname{erf}(\sqrt{-i t \omega}) \\
\left.\left.\left.+e^{2 i t \omega} \sqrt{i a \omega} \operatorname{erf}(\sqrt{i t \omega})\right\}\right]\right] .
\end{gathered}
$$

The expression of Nusselt number is given by

$$
\mathrm{Nu}=-\left.\frac{v}{U_{0}\left(T-T_{\infty}\right)} \frac{\partial T^{*}}{\partial y^{*}}\right|_{y^{*}=0}=\sqrt{\frac{\operatorname{Pr}}{\pi t}} .
$$

\section{Limiting Cases}

The following solutions from the literature appear as the limiting cases of our general solutions.

(i) By taking $\gamma \rightarrow \infty$ into (14) and (15), the corresponding solutions for viscous fluid can be obtained as a special case:

$$
\begin{aligned}
& u_{c}(y, t) \\
& =\frac{H(t)}{4} e^{-i \omega t} \\
& \times\left[e^{-y \sqrt{-i \omega}} \operatorname{erf} c\left(\frac{y}{2} \sqrt{\frac{1}{t}}-\sqrt{-i \omega t}\right)\right. \\
& \left.+e^{y \sqrt{-i \omega}} \operatorname{erf} c\left(\frac{y}{2} \sqrt{\frac{1}{t}}+\sqrt{-i \omega t}\right)\right] \\
& +\frac{H(t)}{4} e^{i \omega t}\left[e^{-y \sqrt{i \omega}} \operatorname{erf} c\left(\frac{y}{2} \sqrt{\frac{1}{t}}-\sqrt{i \omega t}\right)\right. \\
& +b\left[\left(t+\frac{y^{2}}{2}\right) \operatorname{erf} c\left(\frac{y}{2} \sqrt{\frac{1}{t}}\right)-y \sqrt{\frac{t}{\pi}} e^{-y^{2} / 4 t}\right] \\
& +b\left[\left(t+\frac{\operatorname{Pr} y^{2}}{2}\right) \operatorname{erf} c\left(\frac{y}{2} \sqrt{\frac{\operatorname{Pr}}{t}}\right)\right. \\
& \left.\quad-y \sqrt{\frac{1}{\operatorname{Pr}}} \sqrt{\frac{t}{\pi}} e^{-\operatorname{Pr} y^{2} / 4 t}\right],
\end{aligned}
$$




$$
\begin{aligned}
& u_{s}(y, t) \\
& =-\frac{1}{4 i} e^{-i \omega t} \\
& \times\left[e^{-y \sqrt{-i \omega}} \operatorname{erf} c\left(\frac{y}{2} \sqrt{\frac{1}{t}}-\sqrt{-i \omega t}\right)\right. \\
& \left.+e^{y \sqrt{-i \omega}} \operatorname{erf} c\left(\frac{y}{2} \sqrt{\frac{1}{t}}+\sqrt{-i \omega t}\right)\right] \\
& +\frac{1}{4 i} e^{i \omega t}\left[e^{-y \sqrt{i \omega}} \operatorname{erf} c\left(\frac{y}{2} \sqrt{\frac{1}{t}}-\sqrt{i \omega t}\right)\right. \\
& +b\left[\left(t+\frac{y^{2}}{2}\right) \operatorname{erf} c\left(\frac{y}{2} \sqrt{\frac{1}{t}}\right)-y \sqrt{\frac{t}{\pi}} e^{-y^{2} / 4 t}\right] \\
& +b\left[\left(t+\frac{\operatorname{Pr} y^{2}}{2}\right) \operatorname{erf} c\left(\frac{y}{2} \sqrt{\frac{1}{t}}+\sqrt{+i \omega t}\right)\right. \\
& \left.-y \sqrt{\operatorname{Pr}} \sqrt{\frac{t}{\pi}} e^{-\operatorname{Pr} y^{2} / 4 t}\right] .
\end{aligned}
$$

(ii) By taking $\omega=0$, which corresponds to impulsive motion of the plate, then (14) and (17) yield

$$
\begin{aligned}
u_{c}(y, t) & \\
=H(t) & {\left[\operatorname{erf} c\left(\frac{y}{2} \sqrt{\frac{a}{t}}\right)\right] } \\
+ & a b\left[\left(t+\frac{a y^{2}}{2}\right) \operatorname{erf} c\left(\frac{y}{2} \sqrt{\frac{a}{t}}\right)-y \sqrt{a} \sqrt{\frac{t}{\pi}} e^{-a y^{2} / 4 t}\right] \\
+a b & {\left[\left(t+\frac{\operatorname{Pr} y^{2}}{2}\right) \operatorname{erf} c\left(\frac{y}{2} \sqrt{\frac{\operatorname{Pr}}{t}}\right)\right.} \\
& \left.-y \sqrt{\operatorname{Pr}} \sqrt{\frac{t}{\pi}} e^{-\operatorname{Pr} y^{2} / 4 t}\right] ; \quad \operatorname{Pr} \neq 1,
\end{aligned}
$$

$$
\begin{aligned}
& u_{c}(y, t) \\
& =H(t)\left[\operatorname{erf} c\left(\frac{1}{2} \sqrt{\frac{a 1}{t}} y\right)\right] \\
& \quad+\frac{a \operatorname{Gr} y}{2}\left[2 \sqrt{\frac{t}{\pi}} e^{-y^{2} / 4 t}-y \operatorname{erf} c\left(\frac{y}{2 \sqrt{t}}\right)\right] ; \quad \operatorname{Pr}=1,
\end{aligned}
$$

respectively, and (22) and (23) describe the corresponding solution of Stokes' first problem for Casson fluid. It is important to note that exact solutions of Stokes' first problem for Casson fluid (22) and (23) are also not reported in the literature and hence are new. (iii) In this last case, we assume that the flow is induced only due to bounding plate and the corresponding buoyancy forces are zero equivalently; it shows the absence of free convection $(\mathrm{Gr}=0)$ due to the differences in temperature gradient. This shows that the thermal parts of velocities in (14) and (15) are zero. Hence the flow is only governed by the corresponding mechanical parts given by

$$
\begin{gathered}
u_{c}(y, t) \\
=\frac{H(t)}{4} e^{-i \omega t}\left[e^{-y \sqrt{-i \omega a}} \operatorname{erf} c\left(\frac{y}{2} \sqrt{\frac{a}{t}}-\sqrt{-i \omega t}\right)\right. \\
\left.+e^{y \sqrt{-i \omega a}} \operatorname{erf} c\left(\frac{y}{2} \sqrt{\frac{a}{t}}+\sqrt{-i \omega t}\right)\right] \\
+\frac{H(t)}{4} e^{i \omega t}\left[e^{-y \sqrt{i \omega a}} \operatorname{erf} c\left(\frac{y}{2} \sqrt{\frac{a}{t}}-\sqrt{i \omega t}\right)\right. \\
\left.+e^{y \sqrt{i \omega a}} \operatorname{erf} c\left(\frac{y}{2} \sqrt{\frac{a}{t}}+\sqrt{+i \omega t}\right)\right], \\
u_{s}(y, t) \\
=-\frac{1}{4 i} e^{-i \omega t}\left[e^{-y \sqrt{-i \omega a}} \operatorname{erf} c\left(\frac{y}{2} \sqrt{\frac{a}{t}}-\sqrt{-i \omega t}\right)\right. \\
\left.+e^{y \sqrt{-i \omega a}} \operatorname{erf} c\left(\frac{y}{2} \sqrt{\frac{a}{t}}+\sqrt{-i \omega t}\right)\right] \\
+\frac{1}{4 i} e^{i \omega t}\left[e^{-y \sqrt{i \omega a}} \operatorname{erf} c\left(\frac{y}{2} \sqrt{\frac{a}{t}}-\sqrt{i \omega t}\right)\right. \\
\left.+e^{y \sqrt{i \omega a}} \operatorname{erf} c\left(\frac{y}{2} \sqrt{\frac{a}{t}}+\sqrt{+i \omega t}\right)\right] .
\end{gathered}
$$

Note that (24) are identical to those obtained by Fetecau et al. [9]; see (9) and (11). This fact is also shown in Figure 8.

\section{Graphical Results and Discussion}

In this section, the obtained exact solutions are studied numerically in order to determine the effects of several involved parameters such as Prandtl number Pr, Grashof number Gr, Casson parameter $\gamma$, phase angle $\omega t$, and time $t$. Numerical values of skin friction and Nusselt number are computed and presented in tables for different parameters. For the sake of correctness and verification, we have compared our results with those of Fetecau et al. [9]. This comparison is shown in Figure 2. It is found that our limiting solutions (24) are identical to (9) and (11) obtained by Fetecau et al. [9]. This confirms the accuracy of our obtained results.

The velocity profiles for different values of Prandtl number Pr are shown in Figure 3, when the other parameters are fixed. It is observed that velocity of the fluid decreases with increasing Prandtl number. Figure 4 illustrates the profiles of velocity for different values of Gr. It is observed that velocity increases with increasing values of Gr. 




๑๐ Present result _ Fetecau et al. [9]

FIGURE 2: Comparison of the present results (see (24)) with those obtained by Fetecau et al. [9] (see (9) and (11)), when $t=0.2, \omega=0$, $a=1, U=1$, and $v=1$.

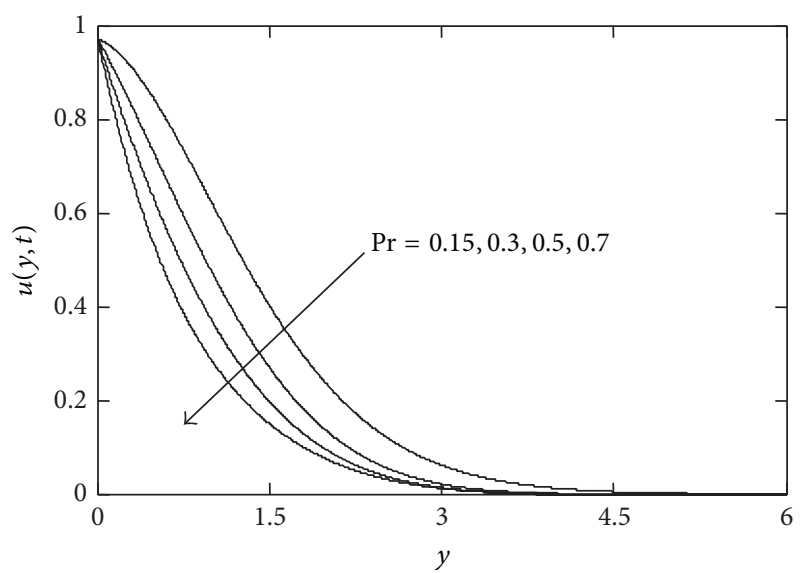

Figure 3: Profiles of velocity for different values of $\operatorname{Pr}$, when $\omega=$ $\pi / 4, t=0.2$, and $\mathrm{Gr}=3$.

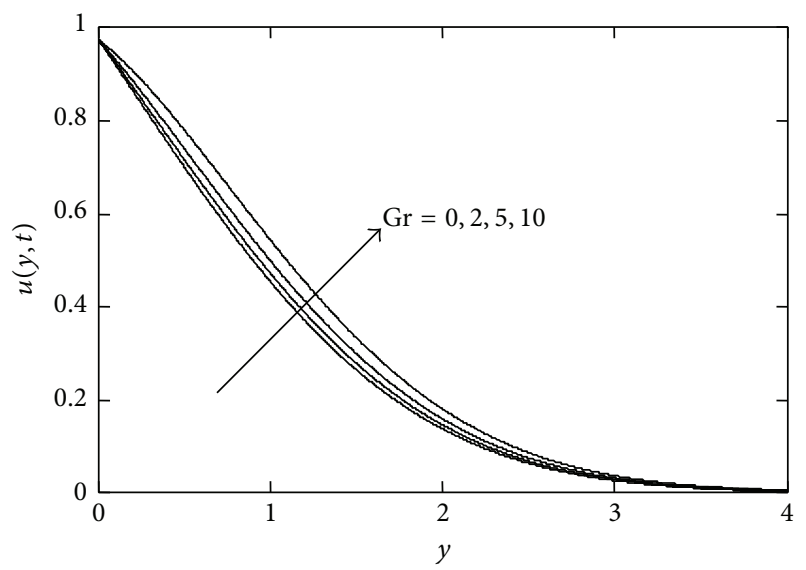

Figure 4: Profiles of velocity for different values of $\mathrm{Gr}$, when $\mathrm{Pr}=$ $0.3, \gamma=0.6, t=0.3$, and $\omega=\pi / 4$.

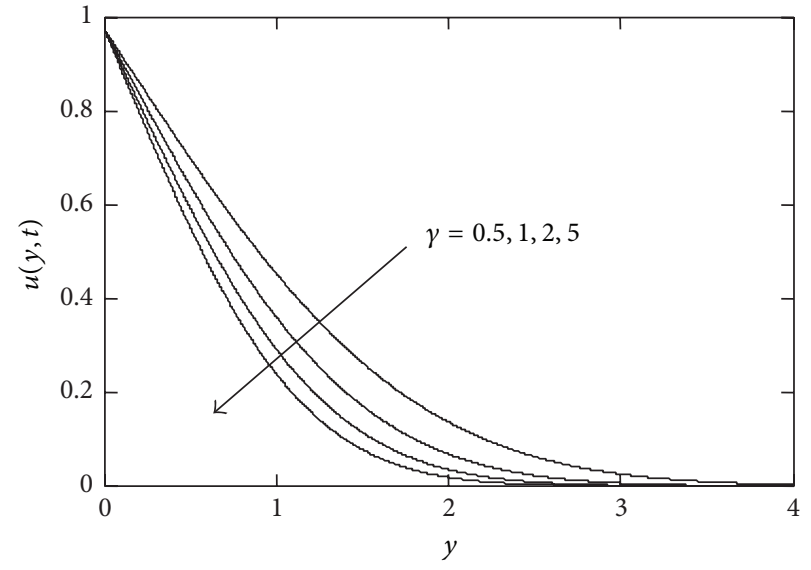

Figure 5: Profiles of velocity for different values of $\gamma$, when $\operatorname{Pr}=$ $0.3, \mathrm{Gr}=0, t=0.3$, and $\omega=\pi / 4$.



FIGURE 6: Profiles of velocity for different values of $\omega t$, when $\mathrm{Gr}=3$, $\operatorname{Pr}=0.3, t=1$, and $\gamma=0.5$.

The influence of Casson fluid parameter on velocity profiles is shown in Figure 5. It is found that velocity decreases with increasing values of $\gamma$. It is important to note that an increase in Casson parameter makes the velocity boundary layer thickness shorter. It is further observed from this graph that when the Casson parameter $\gamma$ is large enough, that is, $\gamma \rightarrow \infty$, the non-Newtonian behaviours disappear and the fluid purely behaves like a Newtonian fluid. Thus, the velocity boundary layer thickness for Casson fluid is larger than the Newtonian fluid. It occurs because of plasticity of Casson fluid. When Casson parameter decreases, the plasticity of the fluid increases, which causes the increment in velocity boundary layer thickness. The graphical results for the phase angle, $\omega t$, are shown in Figure 6. It is observed that the fluid is oscillating between -1 and 1 . These fluctuations near the plate are maximum and decrease for further values of independent variable $y$. This figure can easily help us to check the accuracy of our results. For illustration of such results we have concentrated more on the values of $\omega t=0, \pi / 2$ and $\pi$. We can see that, for these values of $\omega t$, the velocity shows its values either 1,0 , or -1 which are identical with the 


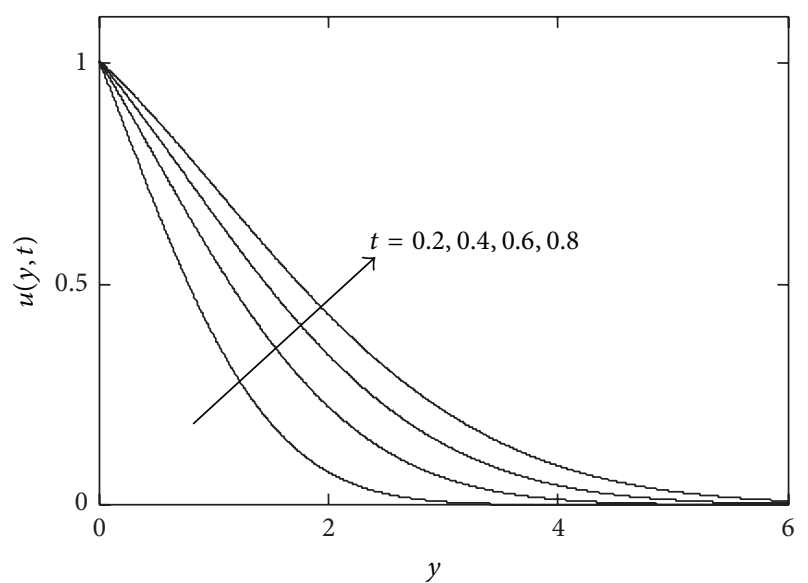

Figure 7: Profiles of velocity for different values of $t$, when $\operatorname{Pr}=$ $0.3, \mathrm{Gr}=3, \gamma=0.5$, and $\omega=0$.

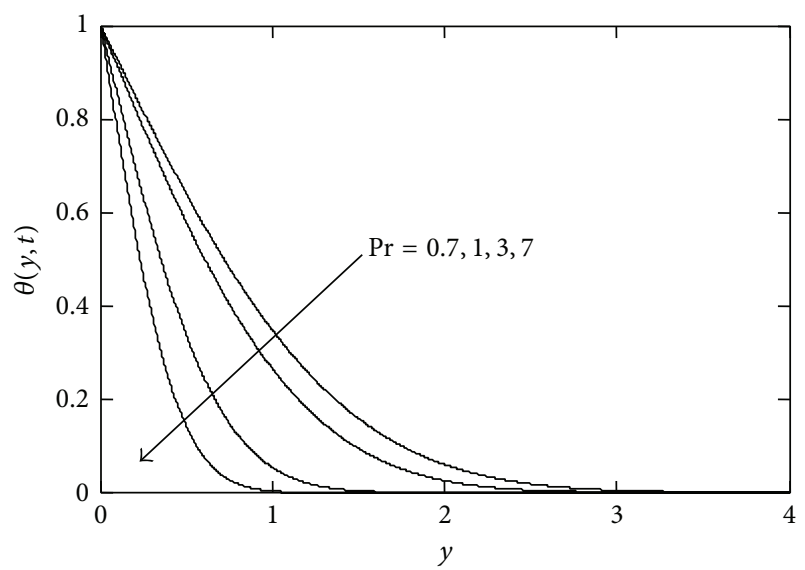

Figure 8: Profiles of temperature for different values of Pr, when $t=0.4$.

imposed boundary conditions of velocity in (11). Hence, both the graphical and mathematical results are found in excellent agreement. In Figure 7 the influence of dimensionless time $t$ on the velocity profiles is shown. It is found that the velocity is an increasing function of time $t$.

It is depicted from Figure 8 that the temperature profile decreases with increasing values of Prandtl number Pr. The specific values of Prandtl number such as $\operatorname{Pr}=0.71, \operatorname{Pr}=1.0$, and $\operatorname{Pr}=7.0$ are important in the sense that, physically, they correspond to air, electrolytic solution, and water, respectively. It is observed that the thermal boundary layer thickness is maximum near the plate and decreases with increasing distance from the leading edge and finally approaches to zero. It is also noticed from this figure that the magnitude of temperature is greater for air compared to electrolytic solution and water. It is justified due to the fact that thermal conductivity of the fluid decreases with increasing Prandtl number Pr and hence decreases the thermal boundary layer thickness and finally the temperature profiles.

Figure 9 is plotted to show the effects of the dimensionless time $t$ on the temperature profiles. Four different values of time $t=0.1, t=0.2, t=0.3$, and $t=0.1$ are

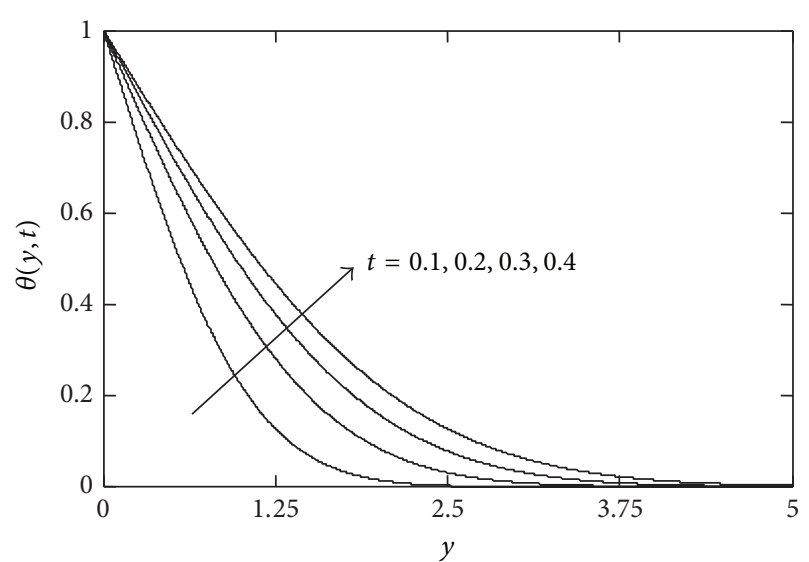

FIGURE 9: Profiles of temperature for different values of $t$, when $\operatorname{Pr}=$ 0.71 .

TABLE 1: Skin friction variations.

\begin{tabular}{lccccc}
\hline $\operatorname{Pr}$ & Gr & $\gamma$ & $\omega t$ & $t$ & $\tau$ \\
\hline 0.3 & 3 & 0.5 & $\pi / 4$ & 0.3 & 3.384043 \\
$\mathbf{0 . 7 1}$ & 3 & 0.5 & $\pi / 4$ & 0.3 & 0.437932 \\
0.3 & $\mathbf{5}$ & 0.5 & $\pi / 4$ & 0.3 & 0.200932 \\
0.3 & 3 & $\mathbf{1 . 0}$ & $\pi / 4$ & 0.3 & 0.536355 \\
0.3 & 3 & 0.5 & $\pi / 2$ & 0.3 & 0.422417 \\
0.3 & 3 & 0.5 & $\pi / 4$ & $\mathbf{0 . 4}$ & 0.307634 \\
\hline
\end{tabular}

TABLE 2: Nusselt number variations.

\begin{tabular}{lcc}
\hline $\operatorname{Pr}$ & $t$ & $\mathrm{Nu}$ \\
\hline 0.3 & 0.3 & 0.564 \\
$\mathbf{0 . 7 1}$ & 0.3 & 0.867 \\
0.3 & $\mathbf{0 . 6}$ & 0.398 \\
\hline
\end{tabular}

chosen. Obviously the temperature increases with increasing time $t$. This graphical behaviour of temperature is in good agreement with the corresponding boundary conditions of temperature profiles as shown in (9). Results for skin friction and Nusselt number are computed in Tables 1 and 2. The computations of skin friction give complex results. Therefore, for the sake of convenience we have considered in Table 1 only its real part. Table 1 shows that skin friction increases with increasing values of $\mathrm{Pr}, \mathrm{Gr}, \gamma$, and $\omega t$, whereas it decreases with increasing values of $t$. On the other hand, it is found from Table 2 that Nusselt number increases with increasing Pr whereas it decreases with increasing $t$.

\section{Conclusion}

In this paper an exact analysis is performed to investigate the unsteady boundary layer flow of a Casson fluid past an oscillating vertical plate with constant wall temperature. The dimensionless governing equations are solved by using the Laplace transform technique. The results for velocity and temperature are obtained and plotted graphically. The numerical results for skin friction and Nusselt number are 
computed in tables. The main conclusions of this study are as follows.

(1) Velocity increases with increasing Gr and $t$, whereas it decreases with increasing values of $\operatorname{Pr}, \gamma$, and $\omega t$.

(2) Temperature increases with increasing $t$, whereas it decreases when Pr is increased.

(3) Skin friction is increasing with increasing values of $\mathrm{Pr}$, $\gamma$, and $\omega t$, whereas it decreases with increasing values of $\mathrm{Gr}$ and $t$.

(4) Nusselt number increases with increasing $\mathrm{Pr}$, whereas it decreases with increasing $t$.

(5) Solutions (24) are found in excellent agreement with those obtained by Fetecau et al. [9].

\section{Conflict of Interests}

The authors declare that there is no conflict of interests regarding the publication of this paper.

\section{Acknowledgments}

The authors would like to acknowledge the SBKWU (HEC), Pakistan, Ministry of Education (MOE), Malaysia, and the Research Management Centre, UTM, for the financial support through vote numbers $06 \mathrm{H} 67$ and $4 \mathrm{~F} 255$ for this research.

\section{References}

[1] M. Sajid, I. Pop, and T. Hayat, "Fully developed mixed convection flow of a viscoelastic fluid between permeable parallel vertical plates," Computers \& Mathematics with Applications, vol. 59, no. 1, pp. 493-498, 2010.

[2] I. Khan, K. Fakhar, and S. Sharidan, "Magnetohydrodynamic rotating flow of a generalized Burgers' fluid in a porous medium with Hall current," Transport in Porous Media, vol. 91, no. 1, pp. 49-58, 2012.

[3] M. I. Anwar, I. Khan, S. Shafie, and M. Z. Salleh, "Conjugate effects of heat and mass transfer of nano fluids over a non-linear stretching sheet," International Journal of Physical Sciences, vol. 7, no. 26, pp. 4081-4092, 2012.

[4] M. Khan, S. Nadeem, T. Hayat, and A. M. Siddiqui, "Unsteady motions of a generalized second-grade fluid," Mathematical and Computer Modelling, vol. 41, no. 6-7, pp. 629-637, 2005.

[5] N. Ali, T. Hayat, and S. Asghar, "Peristaltic flow of a Maxwell fluid in a channel with compliant walls," Chaos, Solitons and Fractals, vol. 39, no. 1, pp. 407-416, 2009.

[6] I. Khan, K. Fakhar, and M. Anwar, "Hydromagnetic rotating flows of an oldroyd-B fluid in a porous medium," Special Topics and Reviews in Porous Media, vol. 3, no. 1, pp. 89-95, 2012.

[7] T. Hayat, M. Awais, and A. Alsaedi, "Newtonian heating and magnetohydrodynamic effects in flow of a Jeffery fluid over a radially stretching surface," International Journal of Physical Sciences, vol. 7, pp. 2838-2844, 2012.

[8] F. Ali, I. Khan, S. Ul Haq, and S. Shafie, "Influence of thermal radiation on unsteady free convection MHD flow of Brinkman type fluid in a porous medium with Newtonian heating," Mathematical Problems in Engineering, vol. 2013, Article ID 632394, 13 pages, 2013.
[9] C. Fetecau, D. Vieru, and C. Fetecau, "A note on the second problem of Stokes for Newtonian fluids," International Journal of Non-Linear Mechanics, vol. 43, no. 5, pp. 451-457, 2008.

[10] M. E. Erdogan, "A note on an unsteady flow of a viscous fluid due to an oscillating plane wall," International Journal of NonLinear Mechanics, vol. 35, no. 1, pp. 1-6, 2000.

[11] W. A. Khan and A. Aziz, "Natural convection flow of a nanofluid over a vertical plate with uniform surface heat flux," International Journal of Thermal Sciences, vol. 50, no. 7, pp. 12071214, 2011.

[12] C. Fetecau and M. Rana, "General solutions for the unsteady flow of second-grade fluids over an infinite plate that applies arbitrary shear to the fluid," Zeitschrift für Naturforschung A: A Journal of Physical Science, vol. 66, no. 12, pp. 753-759, 2011.

[13] N. Casson, "A flow equation for the pigment oil suspensions of the printing ink type," in Rheology of Disperse Systems, pp. 84102, Pergamon, New York, NY, USA, 1959.

[14] T. Hayat, M. Awais, and M. Sajid, "Mass transfer effects on the unsteady flow of UCM fluid over a stretching sheet," International Journal of Modern Physics B, vol. 25, no. 21, pp. 2863-2878, 2011.

[15] R. K. Dash, K. N. Mehta, and G. Jayaraman, "Casson fluid flow in a pipe filled with a homogeneous porous medium," International Journal of Engineering Science, vol. 34, no. 10, pp. 1145-1156, 1996.

[16] Y. C. Fung, Biodynamics Circulation, Springer, New York, NY, USA, 1984.

[17] A. G. Fredrickson, Principles and Applications of Rheology, Prentice-Hall, Englewood Cliffs, NJ, USA, 1964.

[18] J. Boyd, J. M. Buick, and S. Green, "Analysis of the Casson and Carreau-Yasuda non-Newtonian blood models in steady and oscillatory flows using the lattice Boltzmann method," Physics of Fluids, vol. 19, no. 9, Article ID 093103, 2007.

[19] A. V. Mernone, J. N. Mazumdar, and S. K. Lucas, "A mathematical study of peristaltic transport of a Casson fluid," Mathematical and Computer Modelling, vol. 35, no. 7-8, pp. 895912, 2002.

[20] M. Mustafa, T. Hayat, I. Pop, and A. Aziz, "Unsteady boundary layer flow of a Casson fluid due to an impulsively started moving flat plate," Heat Transfer-Asian Research, vol. 40, no. 6, pp. 563576, 2011.

[21] T. Hayat, S. A. Shehzad, A. Alsaedi, and M. S. Alhothuali, "Mixed convection stagnation point flow of casson fluid with convective boundary conditions," Chinese Physics Letters, vol. 29, no. 11, Article ID 114704, 2012.

[22] S. Mukhopadhyay, "Effects of thermal radiation on Casson fluid flow and heat transfer over an unsteady stretching surface subjected to suction/blowing," Chinese Physics B, vol. 22, no. 11, Article ID 114702, 2013.

[23] S. Mukhopadhyay, P. R. De, K. Bhattacharyya, and G. C. Layek, "Casson fluid flow over an unsteady stretching surface," Ain Shams Engineering Journal, vol. 4, no. 4, pp. 933-938, 2013.

[24] K. Bhattacharyya, "Boundary layer stagnation-point flow of casson fluid and heat transfer towards a shrinking/stretching sheet," Frontiers in Heat and Mass Transfer, vol. 4, no. 2, Article ID 023003, 2013.

[25] S. Pramanik, "Casson fluid flow and heat transfer past an exponentially porous stretching surface in presence of thermal radiation," Ain Shams Engineering Journal, vol. 5, no. 1, pp. 205212, 2014.

[26] S. Kim, Study of non-Newtonian viscosity and yield stress of blood in a scanning capillary-tube rheometer (A) [Ph.D. thesis], Mechanical Engineering and Mechanics, 2002. 


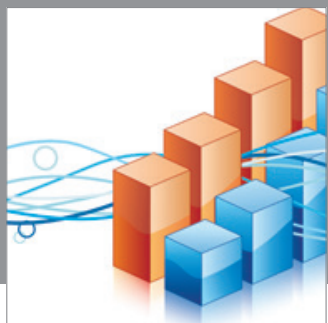

Advances in

Operations Research

mansans

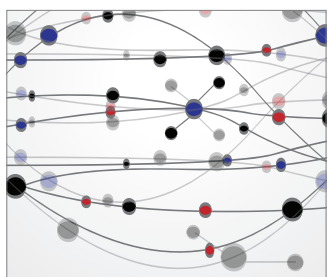

The Scientific World Journal
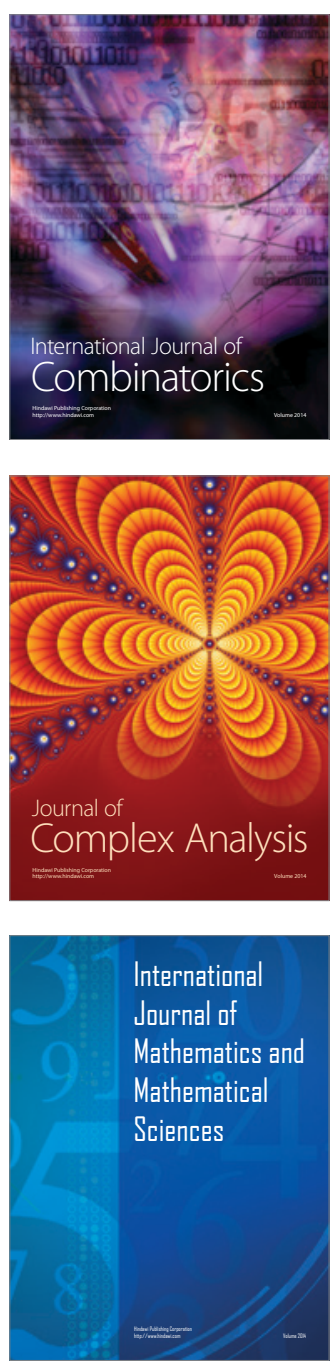


Submit your manuscripts at http://www.hindawi.com
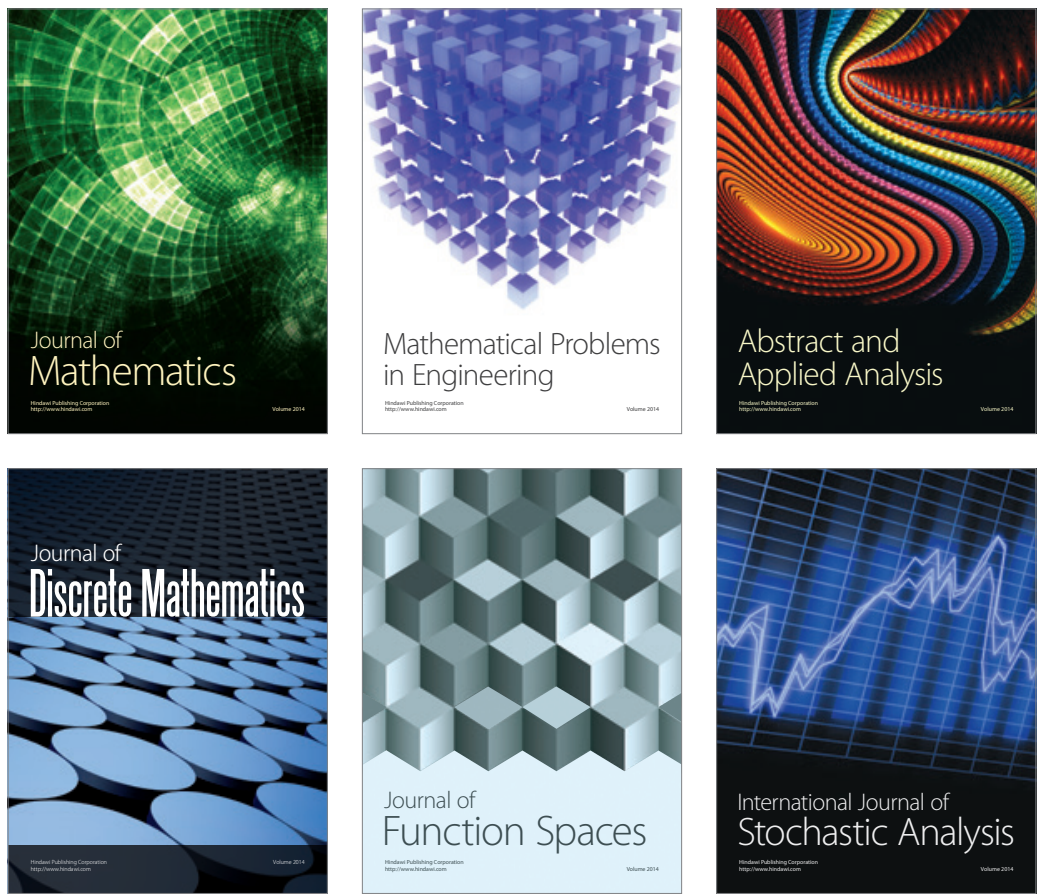

Journal of

Function Spaces

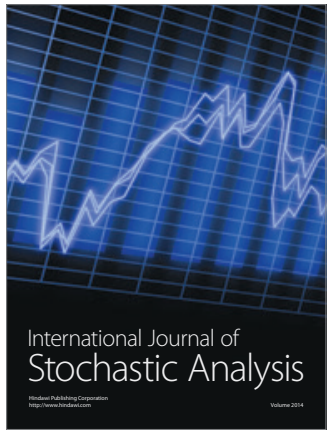

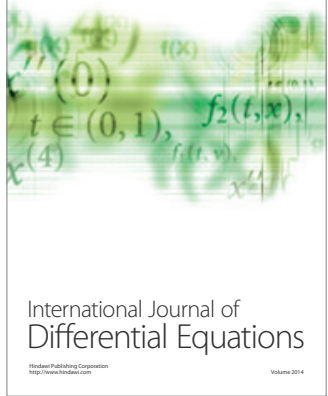
